\title{
NOTES
}

\section{Screen blanking on the Apple IIe}

\author{
BARRY GORDON and BRUCE FIELD \\ The Johns Hopkins University, Baltimore, Maryland
}

Screen blanking on the Apple IIe can be controlled by software after an annunciator output is connected to the chip enable line (Pin 18) of the character generator (UF4). Using Annunciator 3 gives the same functional results with the Apple IIe as does Reed's (1979) hardware modification for the Apple II and Apple $\mathrm{II}+$.

The Apple II series of microcomputers has been widely adapted for experimental work in psychology. Reed (1979) described two hardware modifications enabling precise control of the screen display: sensing the video sync signal so that stimulus and response timing could be synchronized with the display, and screen blanking so that time-consuming or distracting screen operations could be hidden from the subject. The Apple II and II + now have been replaced by the more sophisticated Apple Ile. In the Apple IIe, video sync can be sensed in software without hardware modifications (Apple Ile Reference Manual, p. 154). Also, because of hardware changes, Reed's (1979) screen blanking modification is no longer applicable.

Screen blanking can still be accomplished, however, by disabling the output of the character generator through a modification similar to Reed's and having nearly identical functional results. The screen generator chip (UF4 in Figure 7-13c, Apple Ile Reference Manual, p. 181) has a chip enable line (Pin 18) which can be brought under control in two electrically equivalent ways. Annunciator 2 output can be connected to the line by a jumper wire across Pad X3, one of a set of pads on the right side of the Apple circuit board near the game controller socket. This connection is simple and direct, but it will void the warranty, because it cannot be undetectably reversed. Also, the modification is incompatible with software set up for Reed's (1979) original scheme, which used Annunciator 3. A reversible method to connect any of the annunciator outputs to Pin 18 of UF4 is to insert a fine piece

We thank an anonymous reviewer for making helpful suggestions. Address reprint requests to B. Gordon, Cognitive Neurology, Meyer 2-222, The Johns Hopkins Hospital, 600 N. Wolfe Street, Baltimore, MD 21205. of wire (e.g., No. 22 gauge wirewrap wire) bared at both ends, into the UF4 Pin 18 socket and then into the pin socket of the desired annunciator on the 16-pin game controller socket (see Apple Ile Reference Manual, Table 211, p. 38). Replacing the chip holds the wire in place at one end; the 16-pin game controller plug holds it at the other. This modification allows use of Annunciator 3 output for video blanking, as in Reed's (1979) original suggestion. A small switch placed in series in this wire allows the revision to be enabled or disabled as necessary.

When the Apple Ile is booted or reset, Annunciators 2 and 3 are set to "off" (TTL low) and 0 and 1 are "on" (TTL high). (Note that the Apple Ile Reference Manual is inconsistent in labeling TTL high and low as "on" and "off"; we have adopted the usual convention that TTL high [+3.5 V] is "on.") The opposite state can be toggled in software by PEEKing or POKEing the appropriate memory address (see Table 2-11, p. 38). TTL "on" disables the character generator and blanks the screen; TTL "off' keeps the character generator enabled. Therefore, with this modification, TTL "off" (the initial state for Annunciators 2 and 3) keeps the screen on, while TTL "on" turns off the screen (blanks it).

One potential problem with this Apple Ile modification is not a problem with Reed's Apple II or II + modification: The keyboard input is scrambled or even falsely present while the screen is turned off. This should not be a problem for most applications. If it is, but no keyboard input is necessary, the keyboard buffer can be cleared by software. If input from the keyboard is necessary, the keyboard buffer can first be cleared by software. If input from the keyboard is necessary at all times, then filing Connector X2 open and closing X1 (by jumper wire; see Figure 7-14B, Apple IIe Reference Manual, p. 180) should isolate the keyboard circuitry from the blanking modification. By using jumper wires with switches across both, the original connections can be restored when desirable. This modification cannot be undetectably reversed.

\section{REFERENCES}

Apple Ile reference manual (1982). Cupertino, CA: Apple Computer, Inc.

ReED, A. V. (1979). Microcomputer display timing: Problems and solutions. Behavior Research Methods \& Instrumentation, 11, 572-576.

(Revision accepted for publication May 8, 1985.) 